Reprod. Nutr. Dévelop., 1987, 27 (1 B), 279-280.

\title{
Influence du niveau d'alimentation, de la supplémentation et de la qualité de l'herbe sur la digestibilité en vue de la mesure de l'ingestion au pâturage (') \\ Nicole BARTIAUX-THILL, A. THÉWIS $(*)$, F. RODRIGUEZ $(*)$, E. FRANÇOIS \\ Centre de Recherches Agronomiques de l'Etat, \\ Avenue de la Faculté d'Agronomie, 22, B5800 Gembloux, Belgique. \\ (*) Faculté des Sciences Agronomiques de l'Etat, \\ Chaire de Zootechnie, Passage des Déportés, 2, 85800 Gembloux, Belgique.
}

Summary. There was no relationship between intake level and digestibility with the two grasses studied. Barley decreased total nitrogen and fibre digestibility; sugar beet pulp increased organic matter and fibre digestibility. The digestibilities observed in the pastures were similar to those measured in the stalls.

Deux études ont été effectuées simultanément en cages et au pâturage, sur béliers adultes, recevant de l'herbe de prairie naturelle. En cage, nous avons étudié deux facteurs susceptibles de modifier la digestibilité de l'herbe : 1) le niveau d'alimentation (NA) bien que, pour des herbes de digestibilité élevée, il n'aurait pas d'influence sur la digestibilité (Hutton and Jury, 1964);2) l'apport d'un concentré énergétique (orge ou pulpe) puisque les interactions entre fourrage et concentré dépendent à la fois de la nature du concentré et du fourrage (Demarquilly, 1978, résultats non publiés). Au pâturage, nous avons étudié l'influence de la qualité de l'herbe sur son ingestion et sa digestibilité, en comparaison avec les résultats obtenus en stalles.

Matériel et méthodes. Douze béliers adultes, en cages, sont répartis en 4 lots de 3 animaux suivant un schéma en blocs aléatoires complets. Ils reçoivent 4 repas par jour $(6$ h 00,12 h 00,18 h 00 et 24 h 00$)$ d'une ration constituée d'herbe de prairie naturelle en quantité limitée (50 g MS/ $\mathrm{kg} \mathrm{P}^{0,75}$ ) ou ad libitum, complémentée ou non (à raison de $35 \%$ de la MS totale de la ration). Les herbes expérimentées se différencient par le stade d'exploitation $\left(H_{1}\right.$ : montaison; $H_{2}$ : pleine épiaison). La première mesure dure 4 semaines (préparation après sortie de I'hiver), la seconde 2 semaines, la digestibilité et la quantité ingérée étant déterminées les 7 derniers jours. Parallèlement, en prairie, sur les mêmes herbes, 5 béliers dont on veut déterminer l'ingestion, sont équipés de harnais pour mesurer leur excrétion fécale (F). Deux animaux "échantillonneurs " sont munis de canules œsophagiennes. Les bols œsophagiens et les fèces sont récoltés et échantillonnés chaque jour, à $9 \mathrm{~h} 00$ et $17 \mathrm{~h} 00$. Tous les échantillons sont pesés et lyophilisés. La lignine est déterminée par la méthode de Christian (1971). La digestibilité de l'herbe est estimée à partir des régressions de Théwis et al. (1986).

Résultats et discussion. La contamination de l'herbe 1 par la terre $(17 \%$ de cendres contre 9,7\% pour l'herbe 2) explique son ingestion plus faible aussi bien en stalles qu'en prairie. Par contre, sa qualité plus élevée (MAT $=17,6 \%$ au lieu de 11,$0 ; C B=23,7 \%$ au lieu de 28,2 ) explique sa digestibilité plus élevée (tabl. 1).

Influence du NA et de la complémentation. - La digestibilité de la matière organique (dMO) est indépendante du niveau d'alimentation, ce qui s'explique sans doute par les digestibilités élevées des 2 herbes (> $70 \%$ ). Avec l'herbe 1, l'addition d'orge n'augmente pas dMO mais diminue, de 6 à 7 points, la digestibilité des constituants pariétaux. Cet effet résulte des antagonismes entre la digestion de l'amidon et celle des parois végétales. L'addition de pulpes, au contraire, améliore

(1) Travaux subsidiés partiellement par le Fonds de la Recherche Fondamentale Collective Convention 2.4542.84 et par la Communauté Economique Européenne, contrat $n^{\circ}$ TSD.A-189-B(TT). 
dMO vraisemblablement par suite de l'absence d'amidon et de la digestibilité élevée $(85 \%)$ des constituants pariétaux de la pulpe. Avec /'herbe 2, les effets opposés de l'orge et de la pulpe semblent se compenser. En comparant les digestibilités (1) mesurées et (2) attendues (calculées en prenant les digestibilités de l'orge et de la pulpe données par Demarquilly et al., 1978) des rations avec complément on note que, pour l'herbe 1, l'orge induit des interactions (3) négatives et la pulpe des interactions positives. Pour l'herbe 2, aucune des interactions ne diffère significativement de zéro (tabl. 1).

TABL. 1. - Influence du niveau d'alimentation et de la nature du complément sur la digestibilité (d en $\%$ ) et la matière sèche ingérée (MSI en $\mathrm{g} \mathrm{MS/kg} \mathrm{P}^{0,75}$ ) en stalles.

\begin{tabular}{|c|c|c|c|c|c|c|c|c|c|c|c|}
\hline & \multicolumn{7}{|c|}{ Herbe 1} & \multicolumn{4}{|c|}{ Herbe 2} \\
\hline & $\begin{array}{l}\text { Herbe } \\
\text { seule }\end{array}$ & Herk & $\mathrm{be}+$ & orge & Herbe & $e+p$ & pulpes & $\begin{array}{l}\text { Herbe } \\
\text { seule }\end{array}$ & Her & pulpe & $\begin{array}{l}\text { rge } \\
\text { s }\end{array}$ \\
\hline \multicolumn{12}{|l|}{ En quantité limitée } \\
\hline MSI & 47,4 & $\begin{array}{c}49,5 \\
(1)\end{array}$ & (2) & (3) & $\begin{array}{c}46,9 \\
(1)\end{array}$ & $(2)$ & (3) & 53,1 & $\begin{array}{c}52,1 \\
(1)\end{array}$ & (2) & (3) \\
\hline $\begin{array}{l}d \text { matière organique } \\
d \text { matière azotée } \\
d \text { cellulose brute } \\
d \text { lignocellulose (ADF) } \\
d \text { parois (NDF) }\end{array}$ & $\begin{array}{l}80,2 \\
80,5 \\
81,5 \\
81,3 \\
81,0\end{array}$ & $\begin{array}{l}79,5 \\
75,7 \\
73,9 \\
74,1 \\
72,9\end{array}$ & $\begin{array}{l}82,4 \\
80,0 \\
76,6\end{array}$ & $\begin{array}{l}-2,9 \\
-4,2 \\
-2,8\end{array}$ & $\begin{array}{l}82,7 \\
77,2 \\
82,8 \\
83,3 \\
81,7\end{array}$ & $\begin{array}{l}80,9 \\
73,0 \\
80,7\end{array}$ & $\begin{array}{l}+1,8 \\
+4,2 \\
+2,1\end{array}$ & $\begin{array}{l}75,2 \\
71,0 \\
75,9 \\
73,6 \\
71,9\end{array}$ & $\begin{array}{l}77,7 \\
71,0 \\
72,7 \\
72,7 \\
68,8\end{array}$ & $\begin{array}{l}78,3 \\
68,3 \\
75,1\end{array}$ & $\begin{array}{l}-0,6 \\
+2,7 \\
-2,4\end{array}$ \\
\hline \multicolumn{12}{|l|}{ Ad libitum } \\
\hline $\begin{array}{l}\text { MSI } \\
d \text { matière sèche } \\
d \text { matière organique }\end{array}$ & $\begin{array}{l}60,8 \\
74,6 \\
80,9\end{array}$ & & & & & & & $\begin{array}{l}76,0 \\
72,1 \\
74,2 \\
\end{array}$ & & & \\
\hline
\end{tabular}

(1) digestibilités mesurées (2) digestibilités attendues (3) interactions en points.

Estimation de la matière sèche ingérée (MS/) et de la digestibilité au pâturage. Au pâturage l'estimation de MSI dépend de celle de la digestibilité (MSI = F/1-d). Les techniques lignine fécale ou azote fécal ainsi que celle de la teneur en lignine alimentaire mesurée à partir des bols œsophagiens (BO) donnent des résultats identiques. Par contre, à partir des échantillons d'herbe prélevés par fauche (HF) les résultats sont variables. Néanmoins on peut constater que les digestibilités des herbes pâturées sont identiques à celles mesurées en stalles ; la sélection est faible pour des herbes de cette qualité ; il en serait sans doute autrement pour des dMO à l'auge de 65 à $70 \%$ (tabl. 2).

TABL. 2. - Digestibilité (d en \%) et matière sèche ingérée (MSI en g MS/ $\mathrm{kg} \mathrm{P0,75)} \mathrm{au} \mathrm{pâturage} \mathrm{:}$ comparaison de différentes méthodes de prévision.

\begin{tabular}{llllllll}
\hline & & N fécal & $\begin{array}{c}\text { Lignine } \\
\text { fécale }\end{array}$ & $\begin{array}{c}\text { Lignine marqueur (1) } \\
\text { BO (3) }\end{array}$ & HF (4) & Lignine herbe $\left(^{(2)}\right.$ \\
Herbe 1 & MSI & 76 & 77 & 78 & 82 & 79 & 75 \\
& dMS & 74,1 & 74,4 & 74,3 & 75,7 & 74,6 & 73,4 \\
Herbe 2 & MSI & 88 & 87 & 85 & 89 & 88 & 74 \\
& dMS & 72,8 & 72,4 & 71,5 & 73,3 & 72,3 & 67,9
\end{tabular}

(1) rapport : lignine alimentaire/lignine fécale (2) relation entre la concentration en lignine de l'herbe et la dMS (Théwis et al., 1986) (3) lignine dosée à partir des bols œesophagiens (4) lignine dosée à partir d'un échantillon d'herbe prélevé par fauche (HF).

Christian K. R., 1971. Fld. Stn. Rec. Div. Pl. Ind. Csiro (Aust.), 10, 29-38.

Demarquilly C., Andrieu J., Sauvant D., 1978. In : Alimentation des Ruminants, éd. INRA Publ., Versailles, France, 469-538.

Hutton J. B., Jury K. E., 1964. N.Z.J. Agric. Res., 7, 583-595.

Théwis A., François E., Rodriguez F., Bartiaux-Thill N., 1986. Reprod. Nutr. Dévelop., 26. $263-264$. 